\title{
Anti-angiogenic effect of tanshinone IIA involves inhibition of the VEGF/VEGFR2 pathway in vascular endothelial cells
}

\author{
YINGYING XING ${ }^{1,2}$, JIAJIE TU ${ }^{1,2}$, LUFENG ZHENG $^{1,2}$, LE GUO $^{1,2}$ and TAO XI ${ }^{1,2}$ \\ ${ }^{1}$ School of Life Science and Technology, ${ }^{2}$ Jiangsu Key Laboratory of Carcinogenesis and Intervention, \\ China Pharmaceutical University, Nanjing, Jiangsu 210009, P.R. China
}

Received August 12, 2014; Accepted October 2, 2014

DOI: 10.3892/or.2014.3592

\begin{abstract}
Tanshinone IIA (TSA) is one of the major lipophilic components of Salvia miltiorrhiza Bunge reported to exhibit an antitumor effect. The exact intracellular signaling mechanisms involved remain elusive and were therefore the subject of this study. The process of angiogenesis is related to tumor progression, invasion and metastasis and is generally perceived as an indicator of tumor prognosis. Among the most critical factors that induce angiogenesis, the vascular endothelial growth factor (VEGF)/VEGF receptor 2 (VEGFR2) pathway and CD146 (melanoma adhesion molecule) play key roles in this process. This study aimed to demonstrate that TSA has potent anti-angiogenic activity in vitro and ex vivo. Additionally, we evaluated the role of TSA in the VEGF/ VEGFR2 pathway. Through a series of in vitro experiments, we found that TSA has a negative effect on cell proliferation, migration and tube formation of human umbilical vascular endothelial cells. We further showed that TSA can inhibit angiogenesis using chorioallantoic membrane (CAM) and rat aortic ring assays. Furthermore, western blotting demonstrated that TSA effectively suppressed the expression of VEGR2 and CD146. These results suggest that TSA inhibits angiogenesis by downregulation of the VEGF/VEGFR2 pathway.
\end{abstract}

\section{Introduction}

Danshen (Salvia miltiorrhiza Bunge) has been used extensively and historically in China to treat various diseases, including cardiovascular diseases, cerebrovascular diseases and cancer (1). Tanshinone IIA (TSA) (Fig. 1A) is a major monomer of phenanthrenequinones extracted from the root of Salvia miltiorrhiza, which has many activities. The most intensively investigated are anti-oxidant properties and antiinflammatory activities $(2,3)$. Many studies indicate that

Correspondence to: $\mathrm{Dr}$ Tao $\mathrm{Xi}$, School of Life Science and Technology, China Pharmaceutical University, Nanjing, Jiangsu 210009, P.R. China

E-mail: xitao18@hotmail.com

Key words: angiogenesis, tanshinone IIA, human umbilical vascular endothelial cells, kinase insert domain receptor
TSA is linked to the prevention and therapy of various types of cancer cells such as colon cancer cells $(4,5)$ and human lung cancer cells (6). However, few studies have reported the anti-angiogenic activity and hence the underlying molecular mechanism of TSA.

Angiogenesis is the formation of new blood vessels, which generally occurs during various physiological and pathological processes (7), such as embryonic development, tumor progression and metastasis. It is now widely accepted that the growth and development of malignant tumors require angiogenesis (8). Since the inhibition of angiogenesis could also result in the suppression of tumor growth, we investigated the potential mechanism concerning the anti-angiogenic effect of TSA on human umbilical vascular endothelial cells (HUVECs).

Numerous molecular mechanisms are responsible for mediating the changes in the microenvironment during angiogenic cascades. Vascular endothelial growth factor (VEGF) is one of the most significant and specific angiogenesis factors (9), and is a potent angiogenic catalyst secreted by many types of tumor cells. It plays an important part in tumor progression by promoting neovascularization (10). In addition, the CD146 molecule is a biomarker on the vascular endothelium (11). Evidence shows that CD146 is a co-receptor for kinase insert domain receptor (KDR; a type III receptor tyrosine kinase) also known as vascular endothelial growth factor receptor 2 (VEGFR2) and plays a key role in the VEGF/VEGFR2 pathway in regards to angiogenesis and tumor growth $(12,13)$. What is more, matrix metalloproteinases (MMPs) are key players in the remodeling and degradation of the extracellular matrix (ECM) and components of the basement membrane (14). MMPs can promote tumor angiogenesis through their proteolytic action on growth factors, cell adhesion molecules and other bioactive enzymes (15). A previous study showed that the anti-angiogenic effect of TSA involves inhibition of modification of MMP-2/ tissue inhibitor of metalloproteinase-2 (TIMP-2) secretion in vascular endothelial cells (16). Yet, a particular MMP has the capacity to cleave a given matrix component at certain sites to create fragments that can be recognized by specific receptors. It is therefore not unexpected that a certain MMP may act in either a pro-angiogenic or anti-angiogenic capacity in different environments (17). Notably, we noted that there are many studies related to downstream targets of VEGFR2, such as the PI3K/Akt pathway (18) and p38 (19). They all can be regulated by TSA. These observations suggest that TSA is likely 
a natural inhibitor of KDR and CD146. In the present study, we aimed to demonstrate that TSA has potent anti-angiogenic activity in vitro and ex vivo models. Our results showed that TSA inhibits endothelial cell proliferation, migration and tube formation by targeting CD146 and the VEGF/VEGFR2 pathway in vitro. Moreover, the expression of MMP-2/-9 was examined.

\section{Materials and methods}

Cell culture and reagents. Primary HUVECs were isolated from the umbilical vein vascular wall based on a previous method (20). HUVECs were routinely passage in ECM (Life Technologies, Carlsbad, CA, USA) with $5 \% \mathrm{CO}_{2}$ at $37^{\circ} \mathrm{C}$. Only those cells from passage 3 to 8 were used for experiments.

Cell viability and proliferation assay. HUVECs (5x103/well) were plated onto a 96-well culture plate and incubated for $24 \mathrm{~h}$. Different dilutions of TSA were added and further incubated for $48 \mathrm{~h}$. Viable cells were determined by MTT assay using the MTT Cell Proliferation Assay kit (Promega, Madison, WI, USA) according to the manufacturer's instructions. The number of viable cells was presented relative to the untreated control. Meanwhile, HUVECs were seeded (5,000 cells/well) in a 96-well culture plate and incubated for $24 \mathrm{~h}$. The medium was replaced with ECM, containing $1 \%$ fetal bovine serum (FBS), $10 \mathrm{ng} / \mathrm{ml}$ VEGF and various concentrations of TSA $(4,8$ and $12 \mu \mathrm{M})$. After $48 \mathrm{~h}$, relative cell proliferation was determined by the MTT assay. All assays were performed in triplicate wells.

Transwell assay. Endothelial cell migration was assessed using a modified Boyden chamber assay. HUVECs $\left(1 \times 10^{5}\right)$ were plated in Endothelial cell medium containing 1\% FBS in the upper chamber of the Transwell $(8-\mu \mathrm{m}$ PET; Millipore, Germany). Cells were then treated for $30 \mathrm{~min}$ at $37^{\circ} \mathrm{C}$. Endothelial cell medium containing 1\% FBS, $10 \mathrm{ng} / \mathrm{ml}$ VEGF and various concentrations of TSA $(4,8$ and $12 \mu \mathrm{M})$ were added to the lower chamber. Endothelial cell medium containing $1 \%$ FBS was a negative control. After $8 \mathrm{~h}$, the non-migrated cells were removed by a cotton swap, and the migrated cells were stained with crystal violet and examined under a microscope. The number of migrated cells was quantified by counting the cells with $\mathrm{x} 40$ objective. Migration was normalized to the percentage of migration.

Tube formation assay. The ECM gel-induced capillary tube formation assay was used as an in vitro measurement of angiogenesis. Briefly, a 24-well culture plate was coated with $50 \mu \mathrm{M} /$ well ECM gel (Sigma, Japan) and allowed to stand for $30 \mathrm{~min}$ at $37^{\circ} \mathrm{C}$ to form a gel layer. After gel formation, $1 \times 10^{5}$ HUVECs in $0.5 \mathrm{ml}$ of growth medium were seeded to each well along with $10 \mathrm{ng} / \mathrm{ml}$ VEGF. Various dilutions of TSA were added into the wells and incubated for $6 \mathrm{~h}$ at $37^{\circ} \mathrm{C}$ in a humidified atmosphere with $5 \% \mathrm{CO}_{2}$, and the formation of capillary tubes was photographed using an inverted microscope.

Chorioallantoic membrane (CAM) assay. The effect of TSA on ex vivo angiogenesis was determined by the CAM assay.
Briefly, fertilized chick eggs which were incubated for 6 days were used for the experiment and were incubated at $70 \%$ humidity and $37^{\circ} \mathrm{C}$. A window was opened on the top of each egg after 1 day of incubation. The windows were covered with sterile tape and the eggs were returned to the incubator. After an additional 1 day, the transparent tape was cut open and a drug carrier plate with a $15-\mathrm{mm}$ diameter was placed in the chamber. Twenty microliters of each test reagent was added onto the plate. Test substances including TSA (the dose of TSA was 4,8 and $12 \mu \mathrm{M}$, respectively). The eggs were incubated for $48 \mathrm{~h}$ and photographed. Blood vessel density was quantified by counting the number of branching blood vessels. Each experiment was performed three times and represented as a bar diagram.

Rat aortic ring assay. The rat aortic ring assay was used as the ex vitro angiogenesis study model (21). Dorsal aortas from freshly sacrificed Sprague-Dawley rats were extracted in a sterile manner and rinsed in ice cold phosphate-buffered saline (PBS). They were then cut into 1-mm long rings using a surgical blade. Each ring was placed in a collagen pre-coated 96-well plate. Different dilutions of TSA were added to the wells. On day 7 , the rings were analyzed by phase-contrast microscopy, and microvessel outgrowths were quantified (22) and photographed. All experimental protocols were approved by the Ethics Committee for Animal Experimentation of China Pharmaceutical University.

ELISA. A human MMP-2 and MMP-9 ELISA kit (R\&D Systems, Shanghai, China) was used to determine the levels of MMP-2 and MMP-9 in conditioned media collected from HUVECs with or without a $24-\mathrm{h}$ treatment with TSA. The experimental steps were followed as described in the protocol provided by the manufacturer.

Transfection. The VEGF165 expression plasmid pcVEGF165 was constructed in our laboratory. Cells were transfected with the pcVEGF165 using Lipofectamine 2000 (Life Technology) according to the manufacturer's protocol. After $6 \mathrm{~h}$, the medium was replaced with ECM containing 1\% FBS, $100 \mathrm{ng} / \mathrm{ml}$ endothelial cell growth supplement (ECGS) and various concentrations of TSA $(0,4,8$ and $12 \mu \mathrm{M})$. After 24 and $48 \mathrm{~h}$, total cellular RNA and protein were respectively extracted for the next detection.

$q R T-P C R$. Total cellular RNA was extracted using TRIzol reagent (Gibco-BRL, Gaithersburg, MD, USA), according to the manufacturer's protocol. Total RNA was subjected to cDNA synthesis using $\mathrm{M}-\mathrm{MLV}$ reverse transcriptase. The process was performed at $30^{\circ} \mathrm{C}$ for $10 \mathrm{~min}, 42^{\circ} \mathrm{C}$ for $1 \mathrm{~h}$ followed by denaturation at $95^{\circ} \mathrm{C}$ for $2 \mathrm{~min}$. To determine mRNA expression levels, qRT-PCR was performed using the ABI Prism 7500 Sequence Detector (Applied Biosystems, Life Technologies). cDNA templates $(2 \mu \mathrm{l})$ were amplified in a final volume of $20 \mu \mathrm{l}$ containing the SYBR-Green PCR Master Mix and primer. Melt-curve analysis and agarose gel electrophoresis were used to confirm amplicon specificity. The length of the amplified product was confirmed using $2 \%$ agarose gel electrophoresis. Relative quantification was performed using the $2^{-\Delta \Delta \mathrm{Ct}}$ method. GAPDH served as an 
A<smiles>Cc1coc2c1C(=O)C(=O)c1c-2ccc2c1CCCC2(C)C</smiles>
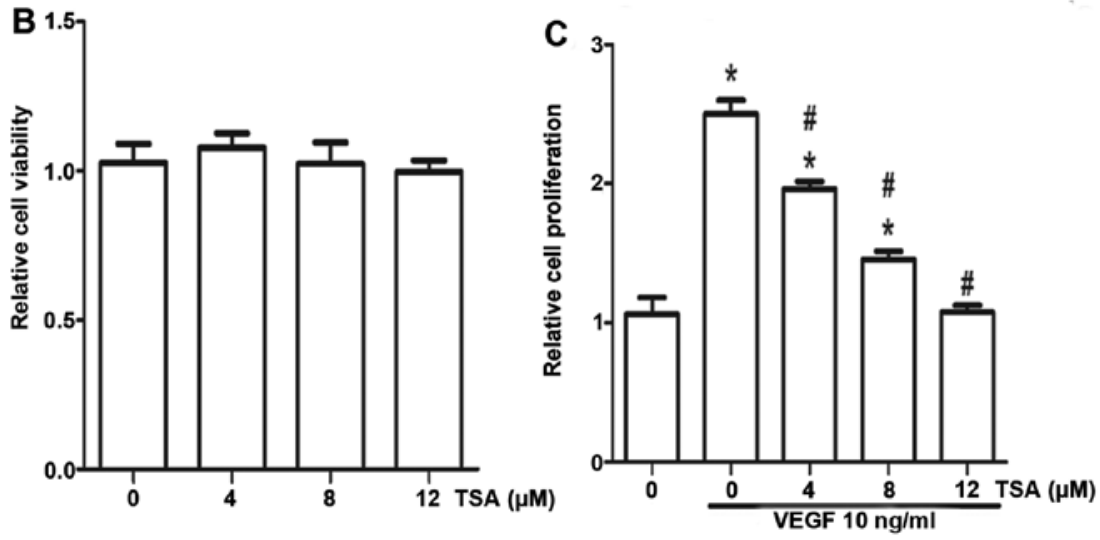

Figure 1. (A) Molecular structure of tanshinone IIA (TSA). (B) Effect of TSA on human umbilical vascular endothelial cell (HUVEC) viability in culture. Cell viability was quantified by MTT assay. (C) TSA inhibits the vascular endothelial growth factor (VEGF)-induced proliferation of endothelial cells. HUVECs were treated with different concentrations of TSA and VEGF for $48 \mathrm{~h}$. Relative cell proliferation was determined by MTT assay. Values are means \pm SD. ${ }^{*} \mathrm{P}<0.05$ denotes a statistically significant difference compared to the untreated controls; ${ }^{*} \mathrm{P}<0.05$ denotes a statistically significant difference compared to the VEGF control. All samples were assayed in triplicate and repeated independently three times.

appropriate reference gene in this experiment. qRT-PCR primers for KDR generated a 371-bp product and were forward, 5'-CTGGCATGGTCTTCTGTGAAGCA-3' and reverse, 5'-CCGCATCACATCCACTGGTATT-3'; primers for CD146 were forward, 5'-TGGTCATCGTGGCTGTGATTGTG-3' and reverse, 5'-CCTTTGGAGGCTTTGGCTGAGAGAA-3'. An alternative set of MMP-2 primers generated a 754-bp product and were forward, 5'-TGACGGTAAGGACGGACTC-3' and reverse, 5'-AGTCCGCCAAATGAACCG-3'; primers for MMP-9 were forward, 5'-TGGGGGGCAACTCGGC-3' and reverse, 5'-GGAATGATCTAAGCCCAG-3'; PCR primers for GAPDH were as described and generated a 450 -bp product forward, 5'-AAGGTCGGAGTCACCGGATT-3' and reverse, 5'-CTGGAAGATGGTGATGGGATT-3'.

Western blotting. Cells were washed twice with $1 \mathrm{X}$ PBS and then lysed in lysis buffer $(20 \mathrm{mM}$ Tris, $150 \mathrm{mM} \mathrm{NaCl}$, $0.25 \%$ NP-40, $1 \mathrm{mM}$ phenylmethylsulfonyl fluoride and $1 \mathrm{X}$ protease inhibitors). Fifty micrograms of protein was analyzed on $8 \%$ SDS-PAGE under denaturing conditions and electrotransferred to PVDF membranes (Millipore, Bedford, MA, USA). Non-specific protein binding was blocked by incubating the membranes with blocking solution (TBST and 10\% non-fat dried milk) for $60 \mathrm{~min}$ at room temperature. Polyclonal antibodies specific for KDR (1:250 in TBST containing 5\% BSA) and CD146 (1:500 in TBST containing 5\% BSA) were applied to the membrane and incubated overnight at $4^{\circ} \mathrm{C}$. Membranes were washed in TBST and then incubated in 1:5,000 diluted goat anti-rabbit IgG-HRP secondary antibody (Bio-Rad,
Hercules, CA, USA) for $1 \mathrm{~h}$ at room temperature. The detection of specific signals was performed using the ECL detection system (Amersham Pharmacia Biotech, Piscataway, NJ, USA). Protein concentrations were measured using the method of Bradford (Bio-Rad).

Statistical analysis. All data were obtained from at least three independent experiments and expressed as mean \pm SD unless stated otherwise. Statistical comparisons were made relative to the negative controls, and the significance was assessed using the Student's t-test and was indicated as $\mathrm{P}<0.05$ or $\mathrm{P}<0.01$ using relevant symbols in the figures and legends. A P-value of $<0.05$ was considered to be statistically significant.

\section{Results}

Effect of tanshinone IIA on the viability and proliferation of HUVECs. Cell viabilty was determined by the MTT assay. Effect of TSA on HUVEC viability in culture is shown in Fig. 1B. TSA was found to be non-toxic to HUVECs at concentrations of 4-12 $\mu \mathrm{M}$ and these concentrations were used for further in vitro study. The proliferation of endothelial cells is important for the formation of new blood vessels. To investigate the mechanism of the anti-angiogenic function of TSA, we performed an MTT assay to explore the effect of TSA on proliferation of endothelial cells. As shown in Fig. 1C, HUVECs showed a very high rate of proliferation when stimulated with VEGF. The endothelial cells were then exposed to TSA of different doses. At $48 \mathrm{~h}$, treatment with TSA $(12 \mu \mathrm{M})$ 


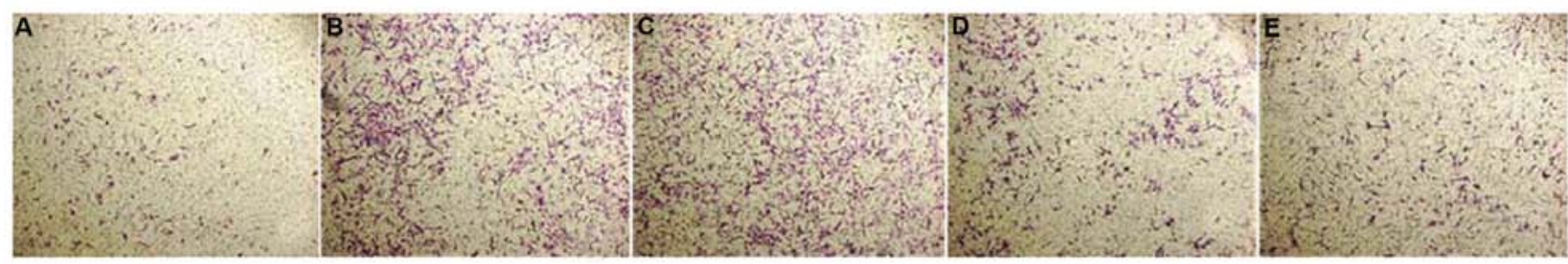

$\mathbf{F}$
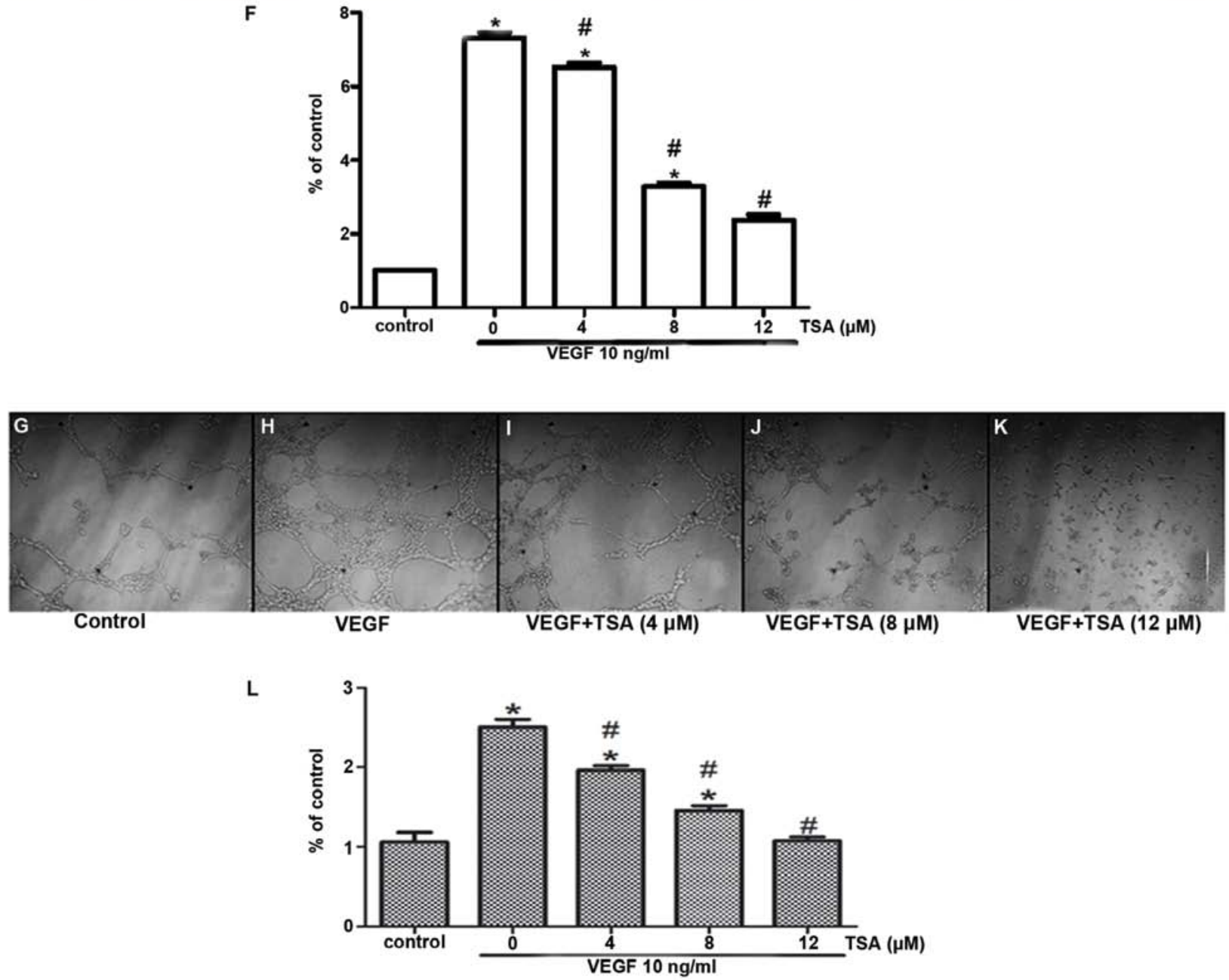

Figure 2. Tanshinone IIA (TSA) inhibits the migration and tube formation of human umbilical vascular endothelial cells (HUVECs). (A-E) The cells on the lower surface of the filter were examined under a contrast microscope (magnification, x100). (F) The inhibitory effect of TSA on HUVEC migration was demonstrated to be dose-dependent. (G-K) Images of tube formation (magnification, $\mathrm{x} 40$ ) were captured. (L) The number of complete tubes in each group was compared and determined. Data are the mean \pm SD calculated from three individual experiments [significance compared with control: " $\mathrm{P}<0.05$ denotes a statistically significant difference compared with the untreated controls and ${ }^{\#} \mathrm{P}<0.05$ denotes a statistically significant difference compared with the vascular endothelial growth factor (VEGF) control].

significantly inhibited the proliferation of HUVECs by $\sim 60 \%$ compared to the untreated cells. This suggests that reduced proliferation contributes to the anti-angiogenic effect of TSA.

Effect of tanshinone IIA on cell migration of HUVECs in vitro. To further understand the anti-angiogenic function of TSA, we examined whether TSA could exert any effect on the migration of HUVECs by Transwell migration assay. The dose-dependent inhibitory effect of TSA on cell migration was demonstrated (Fig. 2A-F). The migration rates of the cells following treatment with 4,8 and $12 \mu \mathrm{M}$ TSA were inhibited by 28,49 and $59 \%$, respectively demonstrating that TSA also dose-dependently inhibits the migration of HUVECs.
Tanshinone IIA inhibits the tube formation of HUVECs in vitro. In order to study the anti-angiogenic function of tanshinone IIA in vitro, we carried out an angiogenesis test by examining the VEGF-induced tube formation of HUVECs. Treatments with TSA $(4,8$ and $12 \mu \mathrm{M})$ inhibited the VEGFinduced tube formation of HUVECs in a dose-dependent manner, which indicates that TSA inhibits the angiogenesis of HUVECs in vitro (Fig. 2G-L).

Tanshinone IIA inhibits the angiogenesis in a chick embryo CAM assay ex vivo. CAM is another ex vivo test for angiogenesis and it is more convenient to quantify vascular development with this method. We placed TSA-impregnated filter disks 


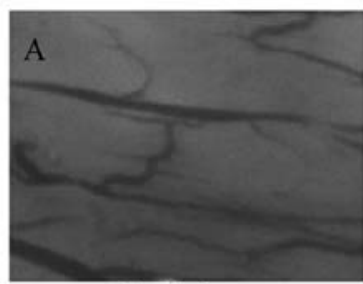

Control

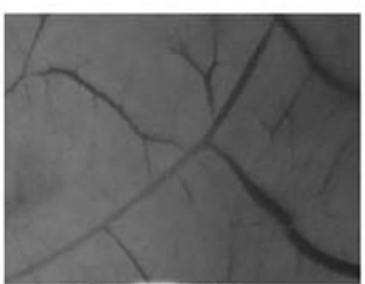

TSA $(4 \mu M)$

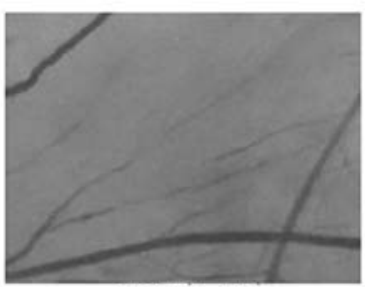

TSA $(8 \mu \mathrm{M})$

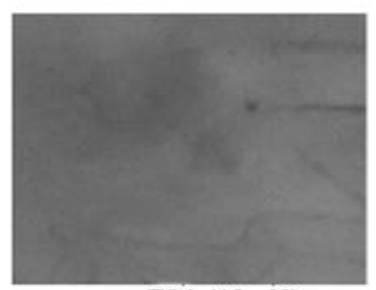

TSA $(12 \mu \mathrm{M})$
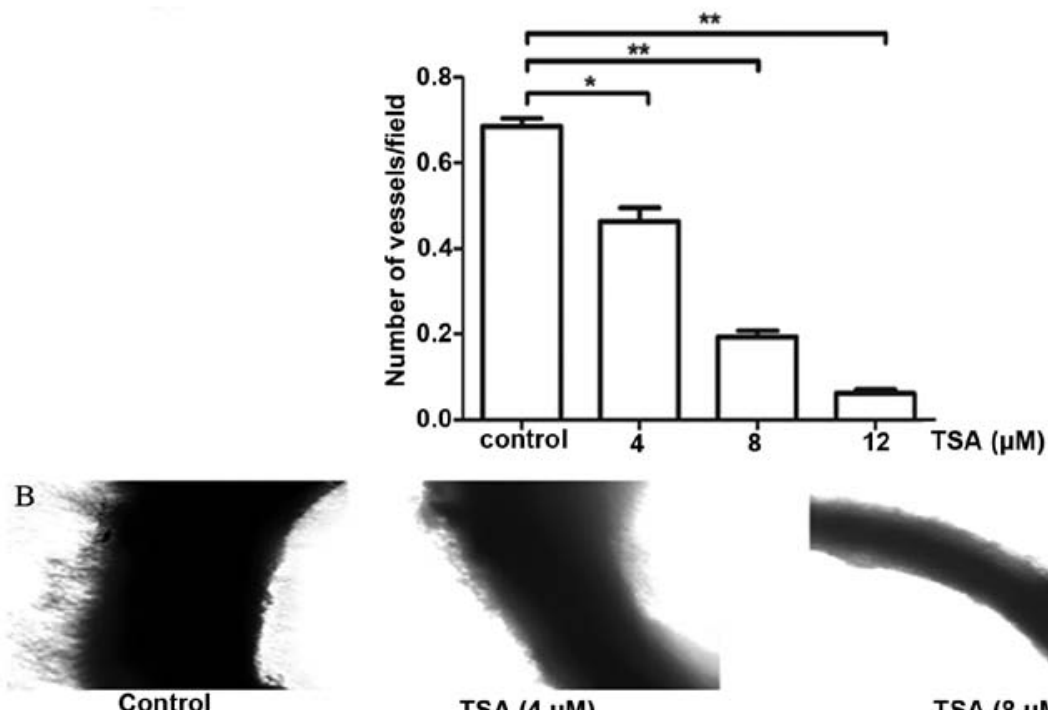

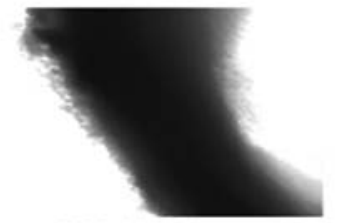

TSA $(4 \mu \mathrm{M})$

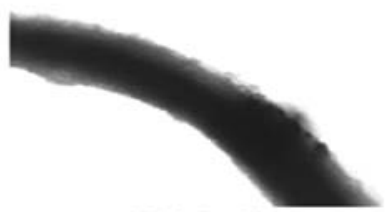

$\operatorname{TSA}(8 \mu \mathrm{M})$

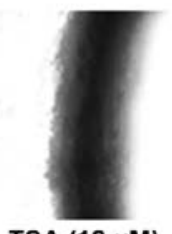

TSA $(12 \mu \mathrm{M})$

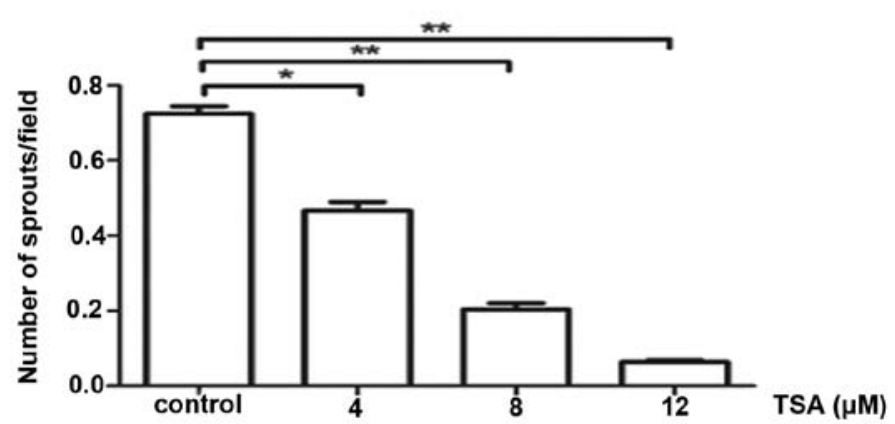

Figure 3. Tanshinone IIA (TSA) inhibits the ex vivo angiogenesis by chorioallantoic membrane (CAM) assay and microvessel outgrowth of the rat aortic ring assay. (A) TSA in DMSO for treatment was gently placed on the CAM. The eggs were incubated for $48 \mathrm{~h}$ and photographed. Blood vessel density was quantified by counting the number of branching blood vessels. (B) Each rat aortic ring was placed in a collagen pre-coated 96-well plate. Vascular endothelial growth factor (VEGF), with or without different dilutions of TSA, was added to the wells. On day 6, the rings were analyzed by phase-contrast microscopy and microvessel outgrowths were quantified and photographed. Each experiment was performed three times and the results are represented as a bar diagram. Significance compared with control, ${ }^{*} \mathrm{P}<0.05$ and ${ }^{* *} \mathrm{P}<0.01$.

on blood vessels in avascular sections of CAM (day 10) for $48 \mathrm{~h}$. The disks and underlying CAM tissues (day 12) were then harvested. We scored angiogenesis by counting the vessel branches present in the CAM tissue below the filter by digital images. Consistent with the data above, upregulation of TSA led to a significant reduction in angiogenesis, while the control CAM exhibited more neovascularization (Fig. 3A), which confirmed the anti-angiogenic potential of TSA through the ex vivo assay.

Tanshinone IIA suppresses the angiogenesis in a rat aortic ring assay ex vitro. We futher explored the anti-angiogenic activity of TSA using ex vitro angiogenesis models. An isolated rat aortic ring was embedded in Matrigel in ECM containing different concentrations of TSA cultured for 7 days. TSA markedly suppressed the outgrowth of cells from the aortic arch in a dose-dependent manner, indicating that TSA inhibits angiogenesis in vitro (Fig. 3B).

Tanshinone IIA inhibits the $m R N A$ and protein expression of $K D R$ and CD146 in HUVECs. VEGF is a critical factor in the process of angiogenesis, and activates the angiogenesis of endothelial cells via integration with its receptor (VEGFR) $(23,24)$. Activation of the VEGF/VEGFR signaling pathway can trigger a network signal cascade, consequently contributing to proliferation, invasion and migration of vascular endothelial cells. Meanwhile, human soluble CD146, a coreceptor for KDR, represents both an attractive biomarker of placental vascular development and a therapeutical target in pregnancy complications associated with pathological 


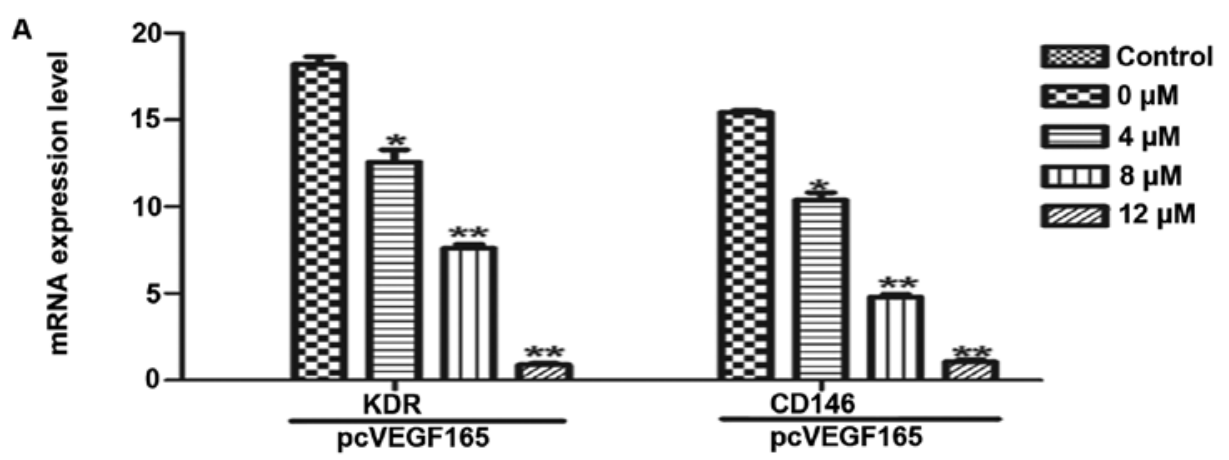

B

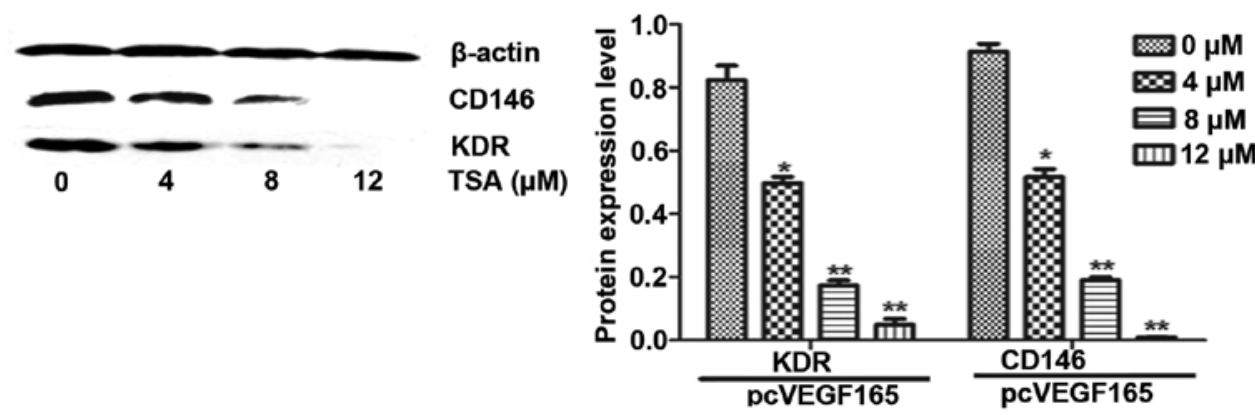

Figure 4. TSA inhibits KDR and CD146 in human umbilical vascular endothelial cells (HUVECs) at the mRNA and protein levels. (A) HUVECs were treated with TSA for $24 \mathrm{~h}$ and the mRNA levels of KDR and CD146 were inhibited as determined by qRT-PCR analysis. (B) After transient transfection of VEGF165, TSA also reduced the protein expression of KDR and CD146 by western blotting. Significance compared with the control, ${ }^{*} \mathrm{P}<0.05$ and ${ }^{* *} \mathrm{P}<0.01$.
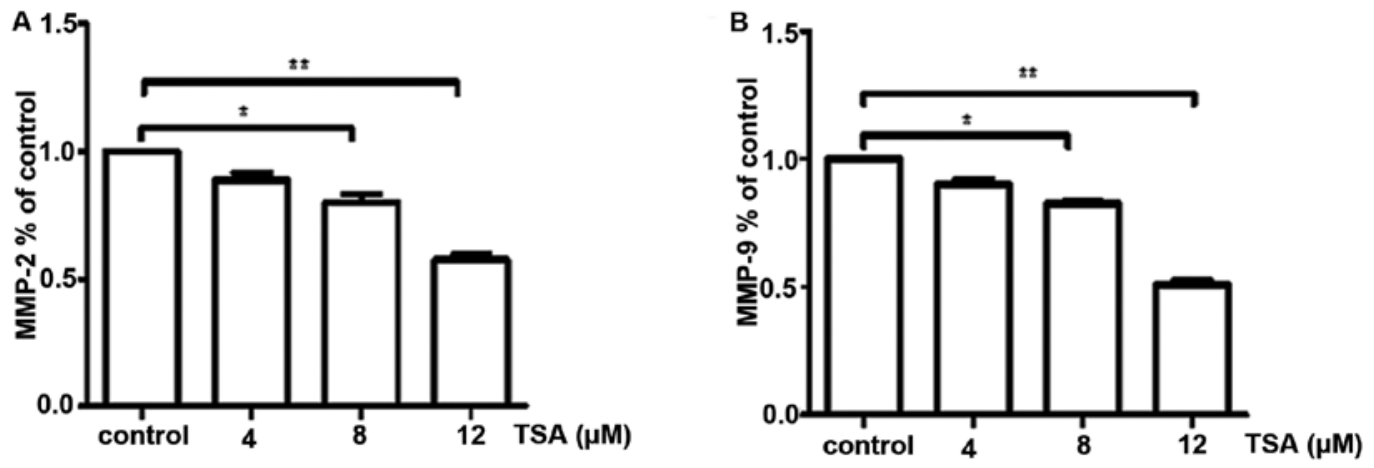

C

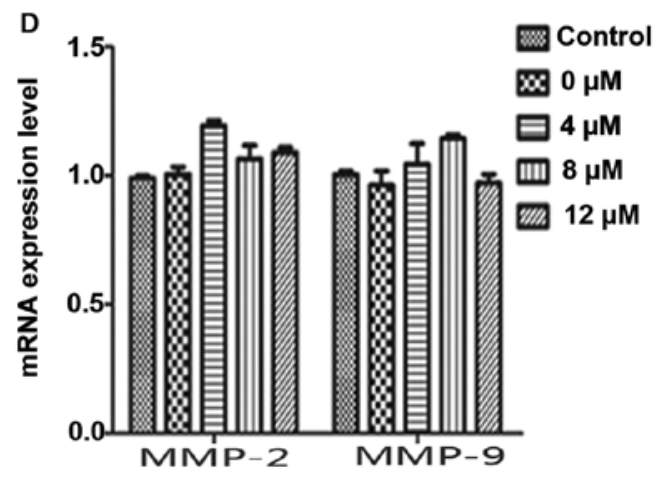

Figure 5. Tanshinone IIA (TSA) reduces matrix metalloproteinase-2 (MMP-2) and MMP-9 expression but not mRNA. (A and B) Human umbilical vascular endothelial cells (HUVECs) treated without or with TSA $(4,8$ and $12 \mu \mathrm{M})$ for $24 \mathrm{~h}$ were processed for ELISA method to analyze the secretion of MMP-2/MMP-9. (C and D) HUVECs were treated with TSA for $24 \mathrm{~h}$ and the mRNA levels of MMP-2, MMP-9 and GAPDH were unchanged as determined by RT-PCR analysis. Significance compared with the control, ${ }^{*} \mathrm{P}<0.05$ and ${ }^{* *} \mathrm{P}<0.01$.

angiogenesis (25). In order to further investigate the mechanism underlying the inhibitory effect of TSA on the invasion of human vascular endothelial cells, we analyzed the effect of TSA on the mRNA and protein expression of KDR and CD146 
by RT-PCR and western blot analysis. HUVECs were transfected with the pcVEGF165 plasmid to induce the expression of KDR. Our results demonstrated the inhibitory effect of TSA on mRNA and protein levels of KDR and CD146 (Fig. 4) in a dose-dependent manner, which definitely indicates that TSA inhibits angiogenesis via repression of the VEGF/VEGFR2 pathway.

Tanshinone IIA reduces MMP-2 and -9 expression but not via $m R N A$. ECM degradation is also important for angiogenesis, and is mainly regulated by a balance between different groups of MMPs (26). MMPs and VEGF/KDR are two foremost factors in angiogenesis (27). To understand the potential mechanism of the inhibitory effect of TSA on the invasion of human vascular endothelial cells through matrix, we analyzed the effect of TSA on the production of MMP-2 and MMP-9 by ELISA method. Our results demonstrated a dose-dependent inhibitory effect of TSA on MMP-2 and MMP-9 (Fig. 5A and B). We further examined whether TSA affects the gene expression of MMP-2 and MMP-9 at the mRNA level. The mRNA levels of MMP-2 and MMP-9 remained unchanged after TSA treatment, which indicates that the anti-angiogenic function of TSA on MMP-2 and MMP-9 in HUVECs is likely mediated by a post-translational mechanism (Fig. 5C and D).

\section{Discussion}

Angiogenesis is critical for the growth and progression of tumors. The importance of angiogenesis to tumor survival offers the possibility of anti-angiogenic therapies in the treatment of cancer (28). TSA has been previously shown to inhibit migration, invasion and metastasis of tumors $(29,30)$. We investigated the molecular mechanism of TSA on angiogenesis in HUVECs. In the present study, we confirmed the inhibitory activity of TSA on angiogenesis. Our results showed that TSA could inhibit various aspects of angiogenesis, including endothelial cell proliferation, migration and the tube formation of HUVECs. Meanwhile, TSA also attenuated ex vivo angiogenesis in a CAM model and microvessel outgrowth in a rat aortic ring assay. These results indicate that TSA has capacity as an anti-angiogenic agent.

Anti-angiogenic drugs have been developed in recent years. Among them the most promising drug for anti-angiogenesis is targeting of the VEGF/VEGFR signaling pathway (31). Since VEGF and its receptors play a critical role in angiogenesis and tumor progression, many approaches have been developed to inhibit this pathway, such as neutralizing antibodies and soluble receptors that inhibit the binding of VEGF to its receptors, antisense constructs against VEGF mRNA, and tyrosine kinase inhibitors that block downstream signaling from membrane-bound VEGF receptors. Although various KDR inhibitors based on this concepts, such as YN968D1, have recently been developed (32), some are still in the clinical stage and have various side effects. Many antibodies and chemical agents that inhibit KDR signaling have been developed and are being tested at the different stages in clinical trials. However, much less is known concerning the inhibitory activity of natural products. Our results revealed a novel activity of TSA inhibition involving the VEGF/VEGFR2 pathway. The next issue that challenged us was how TSA mediated the inhibition of HUVEC proliferation. Physiologically, VEGFR2 (KDR), which is activated by VEGF, undergoes dimerization and ligand-dependent tyrosine phosphorylation in intact cells and results in a mitogenic, chemotactic and pro-survival signal (33). Meanwhile, CD146 is required for VEGF-induced KDR phosphorylation, and AKT/p38 MAPKs/NF- $\mathrm{B}$ activation. Therefore, we examined changes in KDR and CD146 expression levels, which are the starting point of VEGF signaling. Both transcription and translation of KDR and CD146 were downregulated by TSA. This reduction in KDR expression may have been caused by the inhibition of VEGF signaling and the suppression of CD146 by TSA. Evidence suggests that blocking KDR or CD146 limits the ability of most tumors to stimulate the formation of blood vessels $(34,35)$ and the data corroborate the fact that inhibition of KDR and CD146 could suppress angiogenesis.

We attempted to further clarify the potential mechanisms underlying the anti-angiogenic effect of TSA in tumor progression, which have never been studied in detail. It is well known that the angiogenic cascade relies on the degradation of the basement membrane allowing invasion of endothelial cells into the tissue, further removal of obstructing matrix proteins, the migration of endothelial cells and the formation of tubules by endothelial cells to contribute to the chaotic tumor vasculature (36). MMPs, the main group of proteolytic enzymes that degrade and remodel ECM, play a key role in the angiogenic process (37). Therefore, inhibition of the early degradation of ECM proteins predominantly by MMPs is considered to be an important strategy by which to inhibit angiogenesis. In the present study, we found that TSA inhibits the production of MMP-2 and MMP-9. The mRNA levels of MMP-2 and MMP-9 were not altered following TSA treatment, which is in accordance with a previous study (16). Therefore, the antiangiogenic effect of TSA in vascular endothelial cells is also mediated by MMPs.

In summary, TSA was demonstrated to exert in vitro anti-angiogenic effects. For the first time, inhibition of cell migration and invasion was associated with the suppression of the VEGF/VEGFR2 pathway and regulation of MMP-2/-9 secretion in vascular endothelial cells by TSA. The dual inhibitory mechanisms of TSA are thought to contribute to its more effective anti-angiogenic effect. Together with the antitumor effects as identified in previous studies, TSA could be a highly prospective agent with which to inhibit cancer development and tumor angiogenesis. Further studies are needed to evaluate the potential of the anti-angiogenic activity of TSA in tumorigenesis and tumor progression using a carcinogeninduced or genetically engineered tumor model. Collectively, our data indicate that TSA may be a potent inhibitor, antagonizing tumor angiogenesis via the VEGF/VEGFR2 pathway and MMP-2/-9 regulation, and may be a useful agent for the treatment of angiogenesis-related diseases.

In conclusion, our results indicate that TSA inhibits various aspects of angiogenesis, including endothelial cell proliferation, migration and the tube formation of HUVECs. In addition, we demonstrated that TSA inhibits endothelial cell function, at least in part, via the simultaneous inhibition of the VEGF/VEGFR2 signaling pathway and regulation of MMP-2/-9 production. 


\section{References}

1. Bussolino F, Mantovani A and Persico G: Molecular mechanisms of blood vessel formation. Trends Biochem Sci 22: 251-256, 1997

2. Li W, Li J, Ashok M, et al: A cardiovascular drug rescues mice from lethal sepsis by selectively attenuating a late-acting proinflammatory mediator, high mobility group box 1 . J Immunol 178 3856-3864, 2007.

3. Wang AM, Sha SH, Lesniak W and Schacht J: Tanshinone (Salviae miltiorrhizae extract) preparations attenuate aminoglycoside-induced free radical formation in vitro and ototoxicity in vivo. Antimicrob Agents Chemother 47: 1836-1841, 2003.

4. Su CC and Lin YH: Tanshinone IIA downregulates the protein expression of ErbB-2 and upregulates TNF- $\alpha$ in colon cancer cells in vitro and in vivo. Int J Mol Med 22: 847-851, 2008.

5. Su CC: Tanshinone IIA potentiates the efficacy of 5-FU in Colo205 colon cancer cells in vivo through downregulation of P-gp and LC3-II. Exp Ther Med 3: 555-559, 2012.

6. Liu F, Yu G, Wang G, et al: An NQO1-initiated and p53-independent apoptotic pathway determines the anti-tumor effect of tanshinone IIA against non-small cell lung cancer. PLoS One 7: e42138, 2012.

7. Somani RR and Bhanushali UV: Targeting angiogenesis for treatment of human cancer. Indian J Pharm Sci 75: 3-10, 2013.

8. Welti J, Loges S, Dimmeler S and Carmeliet P: Recent molecular discoveries in angiogenesis and antiangiogenic therapies in cancer. J Clin Invest 123: 3190-3200, 2013.

9. Kim KJ, Li B, Winer J, et al: Inhibition of vascular endothelial growth factor-induced angiogenesis suppresses tumour growth in vivo. Nature 362: 841-844, 1993.

10. Plate KH, Breier G, Weich HA and Risau W: Vascular endothelial growth factor is a potential tumour angiogenesis factor in human gliomas in vivo. Nature 359: 845-848, 1992.

11. Kang Y, Wang F, Feng J, Yang D, Yang X and Yan X: Knockdown of CD146 reduces the migration and proliferation of human endothelial cells. Cell Res 16: 313-318, 2006.

12. Jiang T, Zhuang J, Duan H, et al: CD146 is a coreceptor for VEGFR-2 in tumor angiogenesis. Blood 120: 2330-2339, 2012.

13. Wang P, Luo Y, Duan H, et al: MicroRNA 329 suppresses angiogenesis by targeting CD146. Mol Cell Biol 33: 3689-3699, 2013

14. Fanjul-Fernández M, Folgueras AR, Cabrera S and López-Otín C: Matrix metalloproteinases: evolution, gene regulation and functional analysis in mouse models. Biochim Biophys Acta 1803: 3-19, 2010 .

15. Gialeli C, Theocharis AD and Karamanos NK: Roles of matrix metalloproteinases in cancer progression and their pharmacological targeting. FEBS J 278: 16-27, 2011.

16. Tsai MY, Yang RC, Wu HT, Pang JH and Huang ST: Antiangiogenic effect of tanshinone IIA involves inhibition of matrix invasion and modification of MMP-2/TIMP-2 secretion in vascular endothelial cells. Cancer Lett 310: 198-206, 2011.

17. Deryugina EI and Quigley JP: Pleiotropic roles of matrix metalloproteinases in tumor angiogenesis: contrasting, overlapping and compensatory functions. Biochim Biophys Acta 1803: 103-120, 2010.

18. Won SH, Lee HJ, Jeong SJ, et al: Tanshinone IIA induces mitochondria dependent apoptosis in prostate cancer cells in association with an inhibition of phosphoinositide 3-kinase/AKT pathway. Biol Pharm Bull 33: 1828-1834, 2010.
19. Jiao JW and Wen F: Tanshinone IIA acts via p38 MAPK to induce apoptosis and the down-regulation of ERCC1 and lung-resistance protein in cisplatin-resistant ovarian cancer cells. Oncol Rep 25: 781-788, 2011.

20. Baudin B, Bruneel A, Bosselut N and Vaubourdolle M: A protocol for isolation and culture of human umbilical vein endothelial cells. Nat Protoc 2: 481-485, 2007.

21. Burbridge MF and West DC: Rat aortic ring: 3D model of angiogenesis in vitro. Methods Mol Med 46: 185-204, 2001.

22. Yi T, Cho SG, Yi Z, et al: Thymoquinone inhibits tumor angiogenesis and tumor growth through suppressing AKT and extracellular signal-regulated kinase signaling pathways. Mol Cancer Ther 7: 1789-1796, 2008.

23. Cross MJ, Dixelius J, Matsumoto T and Claesson-Welsh L: VEGF-receptor signal transduction. Trends Biochem Sci 28: 488-494, 2003.

24. Guo ZY and Cao BL: Advances of VEGF related molecular promoting tumor angiogenesis and targeting therapy. Zhonghua Bing Li Xue Za Zhi 39: 282-284, 2010 (In Chinese).

25. Kaspi E, Guillet B, Piercecchi-Marti MD, et al: Identification of soluble CD146 as a regulator of trophoblast migration: potential role in placental vascular development. Angiogenesis 16: 329-342, 2013.

26. Hua H, Li M, Luo T, Yin Y and Jiang Y: Matrix metalloproteinases in tumorigenesis: an evolving paradigm. Cell Mol Life Sci 68: 3853-3868, 2011.

27. Zhang JD and Zhang Y: Interregulation of VEGF/R endostatin and MMP in the angiogenesis of hematologic malignancies-review. Zhongguo Shi Yan Xue Ye Xue Za Zhi 13: 1145-1150, 2005 (In Chinese).

28. Carmeliet P and Jain RK: Angiogenesis in cancer and other diseases. Nature 407: 249-257, 2000.

29. Shan YF, Shen X, Xie YK, et al: Inhibitory effects of tanshinone II-A on invasion and metastasis of human colon carcinoma cells. Acta Pharmacol Sin 30: 1537-1542, 2009.

30. Yuxian X, Feng T, Ren L and Zhengcai L: Tanshinone II-A inhibits invasion and metastasis of human hepatocellular carcinoma cells in vitro and in vivo. Tumori 95: 789-795, 2009.

31. Los M, Roodhart JM and Voest EE: Target practice: lessons from phase III trials with bevacizumab and vatalanib in the treatment of advanced colorectal cancer. Oncologist 12: 443-450, 2007.

32. Tian S, Quan H, Xie C, et al: YN968D1 is a novel and selective inhibitor of vascular endothelial growth factor receptor-2 tyrosine kinase with potent activity in vitro and in vivo. Cancer Sci 102: 1374-1380, 2011.

33. Li X, Claesson-Welsh L and Shibuya M: VEGF receptor signal transduction. Methods Enzymol 443: 261-284, 2008.

34. Yan X, Lin Y, Yang D, et al: A novel anti-CD146 monoclonal antibody, AA98, inhibits angiogenesis and tumor growth. Blood 102: 184-191, 2003.

35. Shaheen RM, Davis DW, Liu W, et al: Antiangiogenic therapy targeting the tyrosine kinase receptor for vascular endothelial growth factor receptor inhibits the growth of colon cancer liver metastasis and induces tumor and endothelial cell apoptosis. Cancer Res 59: 5412-5416, 1999.

36. Eguchi M, Masuda $\mathrm{H}$ and Asahara T: Endothelial progenitor cells for postnatal vasculogenesis. Clin Exp Nephrol 11: 18-25, 2007.

37. Packard BZ, Artym VV, Komoriya A and Yamada KM: Direct visualization of protease activity on cells migrating in threedimensions. Matrix Biol 28: 3-10, 2009. 